\title{
NEMATODOS FITOPARÁSITOS ASOCIADOS AL ARROZ EN LAS REGIONES HUETAR NORTE Y HUETAR ATLÁNTICA DE COSTA RICA ${ }^{1}$
}

\author{
Tomás de Jesús Guzmán-Hernández², Silvia Hernández-Villalobos ${ }^{3}$, Ingrid Varela-Benavides ${ }^{3}$, \\ Joaquín Durán-Mora ${ }^{4}$, Wayner Montero-Carmona ${ }^{4}$
}

\begin{abstract}
RESUMEN
Nematodos fitoparásitos asociados al arroz en las regiones Huetar Norte y Huetar Atlántica de Costa Rica. El objetivo de este trabajo fue determinar los nematodos fitoparasitarios asociados al cultivo del arroz en las regiones Huetar Norte y Huetar Atlántica de Costa Rica. Se cuantificó y describió el crecimiento poblacional de los principales géneros mediante modelos matemáticos y se elaboraron tablas de incidencia para los mismos. Un total de catorce campos de cultivo en la región Huetar Norte y quince en la región Huetar Atlántica fueron muestreados durante los años 2006 a 2009. Los nematodos extraídos de suelo y raíz fueron contados e identificados a nivel de género. En la región Huetar Norte el género con mayor densidad de población fue Pratylenchus (17 024 individuos/100 g de raíz) seguido de Meloidogyne (10 343 individuos/100 g de raíz). En la región Huetar Atlántica el género con mayor densidad de población fue Meloidogyne (18 806 individuos/100 g de raíz) seguido de Pratylenchus (8535 individuos/100 g raíz). Otros géneros determinados en las muestras fueron Helicotylenchus, Tylenchorhynchus, Tylenchus y Criconemoides. El modelo matemático que representó mejor la población de Pratylenchus fue el lineal $\left(\mathrm{R}^{2}=0,99\right)$, mientras que el crecimiento poblacional de Meloidogyne se ajustó a un modelo exponencial $\left(\mathrm{R}^{2}=0,91\right)$.
\end{abstract}

Palabras clave: Meloidogyne, Pratylenchus, dinámica poblacional, escalas de evaluación.

\begin{abstract}
Rice plant parasitic nematodes in the Northern and Atlantic Regions of Costa Rica. The objective of this study was to identify the plant parasitic nematodes associated with the rice crop in the Northern and Atlantic regions of Costa Rica. Population growth of the main genuses was described and quantified using mathematical models, for which tables of incidence were constructed. A total of 14 rice fields in the Northern region and 15 rice fields in the Atlantic region were sampled between 2006 and 2009. Nematodes from soil and roots were extracted, then numbers of nematodes of each plant-feeding genus were counted. Pratylenchus was the genus with the greatest population density in the Northern region (17 024 individuals/100 $\mathrm{g}$ of root) followed by Meloidogyne (10 343 individuals/100 $\mathrm{g}$ of root). In the Atlantic Region the genus with the greatest population density was Meloidogyne (18 806 individuals/100 g of root) followed by Pratylenchus (8535 individuals/100 $\mathrm{g}$ of root). Other genera identified in the samples were Helicotylenchus, Tylenchorhynchus, Tylenchus and Criconemoides. The mathematical model that best represented the population of Pratylenchus was linear (R2 = 0.99), while the population growth of Meloidogyne fitted an exponential model $\left(\mathrm{R}^{2}=0.91\right)$
\end{abstract}

Key words: Meloidogyne, Pratylenchus, population dynamics, evaluative scales.

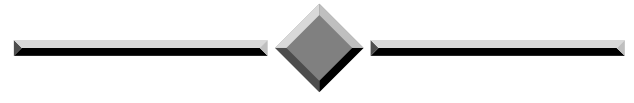

Recibido: 11 de junio, 2010. Aceptado: 16 de mayo, 2011.

2 Director del Doctorado en Ciencias Naturales para el Desarrollo. Instituto Tecnológico de Costa Rica, Sede San Carlos. Apartado 159-7050, Florencia, Alajuela. Tel: (506) 2475-5310. tjguzman@itcr.ac.cr

3 Laboratorio de Nematodos del Instituto Tecnológico de Costa Rica, Sede San Carlos. Apartado 159-7050, Florencia, Alajuela. Tel: (506) 2401-3124. sihernández@itcr.ac.cr; invarela@itcr.ac.cr

4 Instituto Tecnológico de Costa Rica, Sede San Carlos. Apartado 159-7050, Florencia, Alajuela Tel: (506) 2401-3127, 2401-3047 e-mail: jdurán@itcr.ac.cr; wamc.biotec@gmail.com 


\section{INTRODUCCIÓN}

El cultivo del arroz no se encuentra exento de ser atacado por nematodos. A nivel mundial se estima que el $76 \%$ del área dedicada a este cultivo se encuentra infestada con densidades nocivas de nematodos fitoparásitos. Más de 100 especies de estos han sido encontrados en asociación con arroz, inundado y secano; su frecuencia e importancia es muy variable (López et al. 1987).

El arroz es uno de los cereales más importantes en el mundo. En Costa Rica es básico en la alimentación diaria, alcanzando su consumo anual los 55 $\mathrm{kg}$ per cápita. Además la actividad arrocera en Costa Rica es de alta utilidad económica por unidad de área y provee sustento a gran cantidad de familias, de ahí la importancia de los estudios que se realicen en pro del conocimiento y perfeccionamiento del cultivo del arroz (Ramírez 2001).

Observaciones preliminares hechas en plantaciones de arroz de secano en la zona sureste de Costa Rica, demostraron que las mayores densidades poblacionales de nematodos fitoparásitos se localizan en los primeros $15 \mathrm{~cm}$ de profundidad y que, horizontalmente, las densidades variaron significativamente en pequeñas parcelas adyacentes entre sí (López et al. 1987).

Por su parte Sancho y Salazar (1985), elaboraron un trabajo de reconocimiento de los nematodos parásitos del arroz en el sureste de Costa Rica, en el cual Helicotylenchus sp. fue el nematodo más común. Le siguieron en orden descendente Criconemella onoensis, Tylenchorhynchus annulatus, Tylenchus sp, Pratylenchus zeae y Meloidogyne salasi. En lo referente al análisis de raíces, estos investigadores determinaron que únicamente $P$. zeae y $M$. salasi estaban presentes a nivel de raíces. $T$. annulatus tuvo las densidades poblacionales más altas, y Helicotylenchus sp fue la especie más frecuentemente en los campos arroceros del sureste costarricense.

Aunque el aporte más importante de los nematodos que habitan el suelo es mantener la fertilidad del mismo, algunas especies fitófagas causan pérdidas significativas en los cultivos. Los nematodos producen daño mecánico o afectación de los procesos fisiológicos del desarrollo de la planta, disminuye el vigor y reducen la capacidad para soportar situaciones de estrés fisiológico, además producen pequeñas heridas, que luego se infectan con otros patógenos.
El objetivo de este trabajo fue determinar los nematodos fitoparasitarios asociados al cultivo del arroz, en las regiones Huetar Norte y Huetar Atlántica de Costa Rica.

\section{MATERIALES Y MÉTODOS}

Se realizaron muestreos en campos sembrados con arroz, de productores asociados a la Corporación Arrocera Nacional, en las regiones Huetar Norte (catorce productores, $700 \mathrm{ha}$ ) y Huetar Atlántica (quince productores, 140 ha), de Costa Rica. Los muestreos se realizaron entre agosto de 2006 y abril de 2009 en los ciclos de siembra, en intervalos de dos semanas en los años 2006 y 2007, y de cuatro semanas en los años 2008 y 2009.

Cada campo fue dividido en lotes, de acuerdo a la dimensión del mismo, en cada uno se tomó una muestra con la ayuda de una pala (Figura 1), compuesta por cinco puntos de muestreo separados entre sí por 35 $\mathrm{m}$, en forma de zig zag, en cada punto de muestreo se extrajeron las raíces y suelo alrededor de una macolla y a $20 \mathrm{~cm}$ de profundidad. En el caso de que el campo solo estuviera preparado para la siembra, se tomó únicamente la muestra de suelo. El número de lotes y su ubicación en el campo se estimó dependiendo del tamaño y su diversidad de ambientes, de manera que

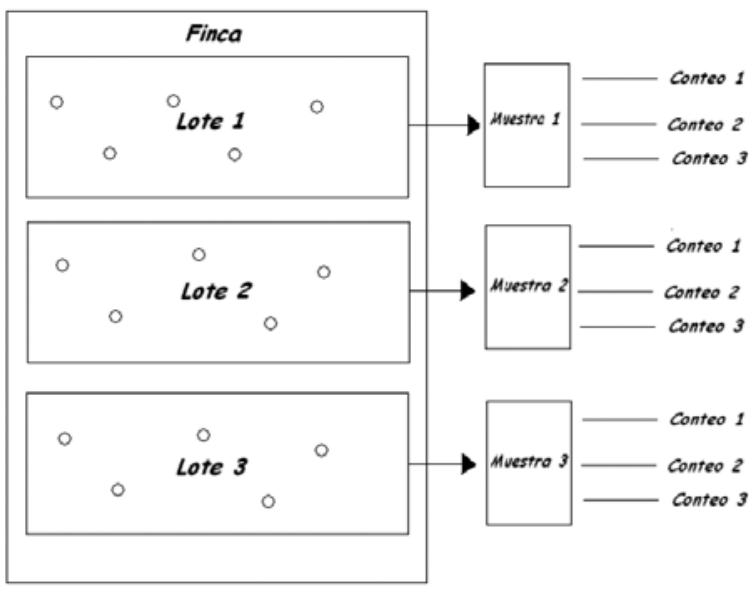

Figura 1. División de los lotes en cada campo y forma de toma de muestras para la extracción de nematodos en raíz y suelo, colectadas en cultivos de arroz en las regiones Huetar Norte y Huetar Atlántica de Costa Rica, en el periodo 2006-2009. 
las muestras representaran la totalidad del campo. En total, se colectaron 234 muestras de raíz y 243 de suelo en la Región Huetar Norte y 186 muestras de raíz y 192 de suelo en la Región Huetar Atlántica.

Luego de su colecta, las muestras fueron rotuladas apropiadamente y colocadas en cajas de aislamiento térmico para su transporte hasta el Laboratorio de Nematología del Instituto Tecnológico de Costa Rica Sede San Carlos, en Santa Clara, Florencia, provincia de Alajuela. Una vez en el laboratorio cada muestra compuesta fue dividida en raíz y suelo. El suelo fue homogenizado, cuarteado y procesado por el método del Embudo de Berman para la extracción de los nematodos. Las raíces fueron lavadas y pesadas, se tomaron $25 \mathrm{~g}$ de raíz que fueron macerados en una licuadora a alta velocidad por quince segundos, los nematodos fueron extraídos por el método de centrifugación - flotación (Esquivel 1996).

Para cada muestra compuesta se procesaron tres submuestras de suelo de $25 \mathrm{~g}$ cada una y tres submuestras de raíz del mismo peso. A cada una de las submuestras de suelo y raíz, se les aplicó el protocolo de extracción correspondiente y posteriormente de este proceso, dichas extracciones fueron colocadas en una cámara para realizar el conteo e identificación a nivel de género de los nematodos fitoparásitos presentes en ella, esto con la ayuda de un microscopio invertido. Se calculó frecuencia de presencia de cada género, densidad promedio por $100 \mathrm{~g}$ de raíz o suelo y la correlación entre la densidad poblacional de nematodos y la edad de la plantación. Los datos fueron sometidos a análisis de regresión y se calcularon correlaciones entre las densidades poblacionales de nematodos y la edad de la plantación en días después de la siembra.

Para la determinación de las escalas empíricas de incidencia se tuvieron en cuenta los resultados obtenidos en los modelos matemáticos de regresión de las dinámicas poblacionales, de todos los productores, los años, las zonas y los ciclos. La determinación sistemática de las poblaciones desde la siembra y hasta la fase final del cultivo, fue el insumo necesario para la confección de dicho instrumento. Para la confección de las escalas se tuvieron en cuenta los nematodos con mayor presencia en las plantaciones de arroz. Por lo que se seleccionaron el Meloidogyne y Pratylenchus.

Los niveles de incidencia que aparecen fueron calculados sobre la base de los modelos matemáticos, y los datos reales de campo, que aparecen en cada región y género, en función de los días de siembra.

\section{RESULTADOS Y DISCUSIÓN}

\section{Frecuencia de presencia y densidad poblacional estimada de los nematodos}

Un total de nueve géneros de nematodos fitoparásitos fueron detectados en las muestras recolectadas en la Región Huetar Norte y diez en la Huetar Atlántica asociados al cultivo del arroz en Costa Rica.

Meloidogyne y Pratylenchus, se presentaron en más del $80 \%$ de las muestras de raíz en ambas regiones. Pratylenchus fue el género que más frecuentemente fue detectado, estando en $99,1 \%$ de las muestras en la Región Huetar Norte y en 97,3\% de la Huetar Atlántica. Meloidogyne, el segundo género en importancia, fue más frecuentemente detectado en las muestras de la Región Huetar Atlántica $(87,6 \%)$ que en las de la Región Huetar Norte (79,1\%). Helicotylenchus también estuvo presente en las muestras con una frecuencia mayor al 50\% (Figura 2).

Meloidogyne y Pratylenchus, también mostraron las mayores densidades estimadas por peso fresco de raíz y suelo, siendo ambas superiores a los 10000 individuos/100 g de raíz, en las dos regiones. El tercer género en importancia fue Helicotylenchus, seguido por Criconemoides, Tylenchorhynchus y Paratylenchus (Cuadro 1). Todos estos han sido reportados como asociados el cultivo del arroz en Costa Rica (González 1978, Sancho y Salazar 1985).

En la región Huetar Norte las densidades máximas detectadas, fueron para el género Pratylenchus (Cuadro 1). El siguiente nematodo en importancia fue

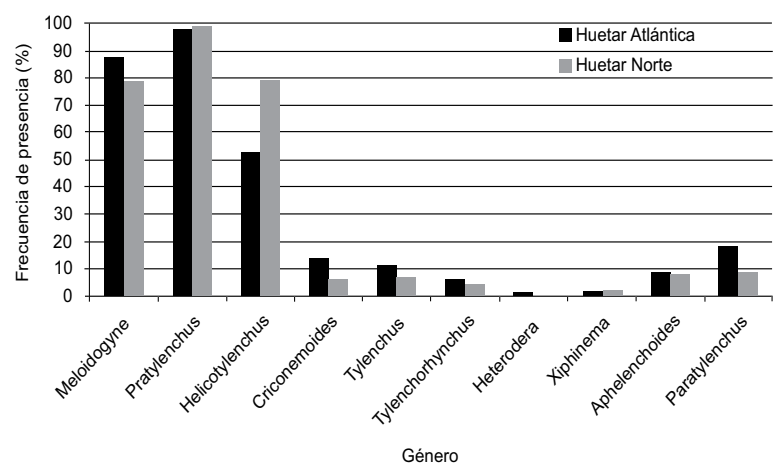

Figura 2. Frecuencia de nematodos fitoparásitos, en muestras de raíz de arroz colectadas en campos de cultivo en las regiones Huetar Norte y Huetar Atlántica de Costa Rica, en el periodo 2006-2009. 
Cuadro 1. Densidad estimada de nematodos fitoparásitos por peso fresco de raíz y suelo, en muestras colectadas en campos de cultivo de arroz en las regiones Huetar Norte y Huetar Atlántica de Costa Rica, en el periodo 2006-2009.

\begin{tabular}{lcc|cc}
\hline & \multicolumn{2}{c|}{ Huetar Norte } & \multicolumn{2}{c}{ Huetar Atlántica } \\
\cline { 2 - 5 } \multicolumn{1}{c}{ Género } & $\begin{array}{c}\text { Individuos en } \\
\text { 100 g de raíz }\end{array}$ & $\begin{array}{l}\text { Individuos en } \\
\mathbf{1 0 0} \text { g de suelo }\end{array}$ & $\begin{array}{c}\text { Individuos en } \\
\text { 100 g de raíz }\end{array}$ & $\begin{array}{c}\text { Individuos en } \\
\mathbf{1 0 0} \text { g de suelo }\end{array}$ \\
\hline Meloidogyne & 18807 & 18 & 10344 & 10 \\
Pratylenchus & 8535 & 15 & 17024 & 31 \\
Helicotylenchus & 98 & 10 & 345 & 25 \\
Criconemoides & 3 & 0 & 1 & 0 \\
Tylenchus & 8 & 2 & 7 & 1 \\
Tylenchorrhynchus & 12 & 0 & 11 & 1 \\
Heterodera & 5 & 0 & 5 & 0 \\
Xiphinema & 2 & 0 & 2 & 0 \\
Aphelenchoides & 16 & 0 & 362 & 1 \\
Paratylenchus & 223 & 0 & 205 & 0 \\
Rotylenchus & 0 & 0 & 0 & 0 \\
Longidorus & 0 & 0 & 6 & 0 \\
Vida libre & 3282 & 171 & 2822 & 105 \\
\hline
\end{tabular}

Meloidogyne que presentó densidades estimadas de 10344 individuos/100 g de raíz. La predominancia de Pratylenchus en los campos de arroz en la Región Huetar Norte ya había sido reportada por López (2006). La importancia de un manejo integrado de Pratylenchus se hace más evidente si se toma en cuenta que puede sobrevivir en el suelo hasta seis meses en barbecho (Suárez y Rosales 2004).

Por su parte, en la región Huetar Atlántica, Meloidogyne fue el nematodo que se encontró en mayor densidad (Cuadro 1). Este nematodo endoparásito sedentario es muy común en las regiones tropicales, y que ataca gran variedad de cultivos. Según Sancho et al. (1987) el género Meloidogyne podría ser considerado el más importante en las plantaciones de arroz en el Pacífico Sureste.

Los resultados mostrados en el Cuadro 1 sugieren que en la Región Huetar Norte, Pratylenchus predomina sobre Meloidogyne y en la Región Huetar Atlántica el comportamiento es inverso. Umesh (1994) en un experimento efectuado en Cebada, define la competencia entre las especies $P$. negletus y $M$. chitwoodi, indicando que la especie que parasitó primero las raíces impidió la penetración y desarrollo de la otra. Fernández y Ortega (1982) explican que este efecto se debe al daño que causa en los tejidos el género que invade en primera instancia.
Con respecto a la densidad estimada de los nematodos fitoparásitos en las muestras de suelo, la tendencia es similar a la presentada en raíz. Aunque, Helicotylenchus, que es un nematodo ectoparásito, tiene mayor importancia relativa en suelo.

Con respecto a otros géneros, los mismos fueron detectados en densidades poblacionales bajas (Cuadro 1), y se presentaron en pocos muestreos (Figura 2). Tylenchus, Heterodera, Rotylenchus y Paratylenchus, no habían sido reportados como asociados al cultivo del arroz en Costa Rica.

Los nematodos de vida libre presentaron las densidades poblacionales estimadas más altas en el suelo (Cuadro 1), hay que apuntar que dentro del grupo de los nematodos se agrupan más de 15000 especies y que la mayor parte de las infestaciones patógenas causadas por nematodos pertenecen a unas cuantas especies de nematodos fitófagos.

\section{Dinámica poblacional de Pratylenchus y Meloidogy- ne en plantaciones de arroz de las Regiones Huetar Norte y Huetar Atlántica de Costa Rica}

La densidad poblacional del género Pratylenchus mostró una correlación alta (mayor a 0,9 ) con respecto a la edad de la plantación de arroz, tanto en la Región 
Huetar Norte como en la Región Huetar Atlántica (Figuras 3 y 4), este incremento puede explicarse con una función de regresión lineal, la cual permitiría eventualmente prever el grado de infección realizando únicamente algunos muestreos. Esta descripción de la dinámica poblacional de Pratylenchus resulta muy importante si se quiere realizar un manejo integrado para su control.

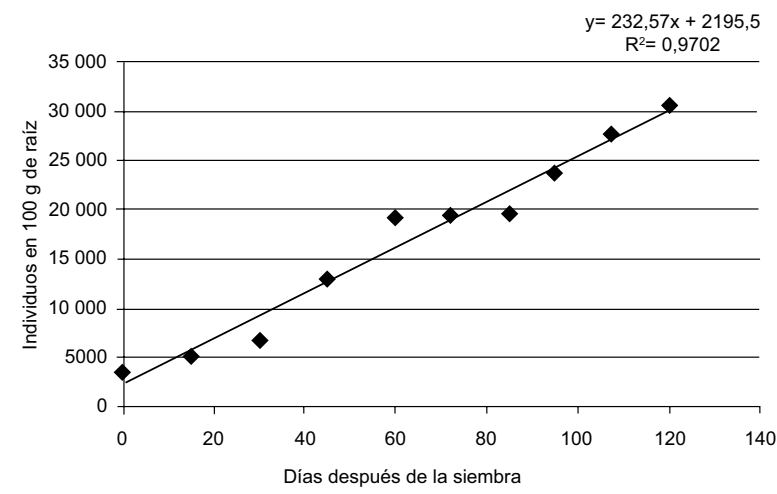

Figura 3. Progresión poblacional de Pratylenchus en raíz de arroz según edad de la plantación en muestras colectadas en la región Huetar Norte de Costa Rica, en el periodo 2006-2009.

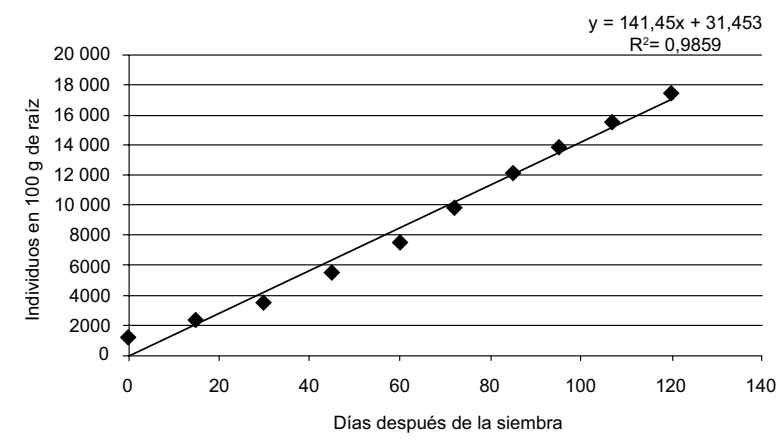

Figura 4. Progresión poblacional de Pratylenchus en raíz de arroz según edad de la plantación en muestras colectadas en la región Huetar Atlántica de Costa Rica, en el periodo 2006-2009.

La densidad poblacional de Meloidogyne tuvo una correlación alta con respecto a la edad de la plantación de arroz, tanto en la Región Huetar Norte como en la Región Huetar Atlántica (Figuras 5 y 6). Sin embargo,

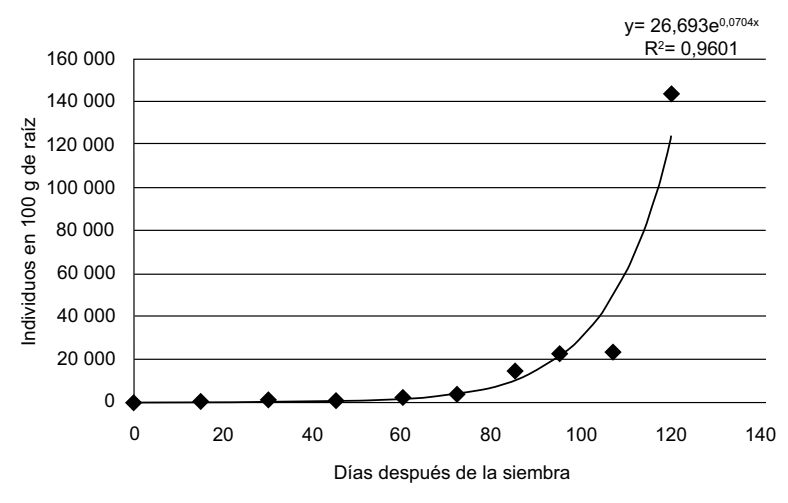

Figura 5. Progresión poblacional de Meloidogyne en raíz de arroz según edad de la plantación en la Región Huetar Norte, Costa Rica, en el periodo 2006-2009.

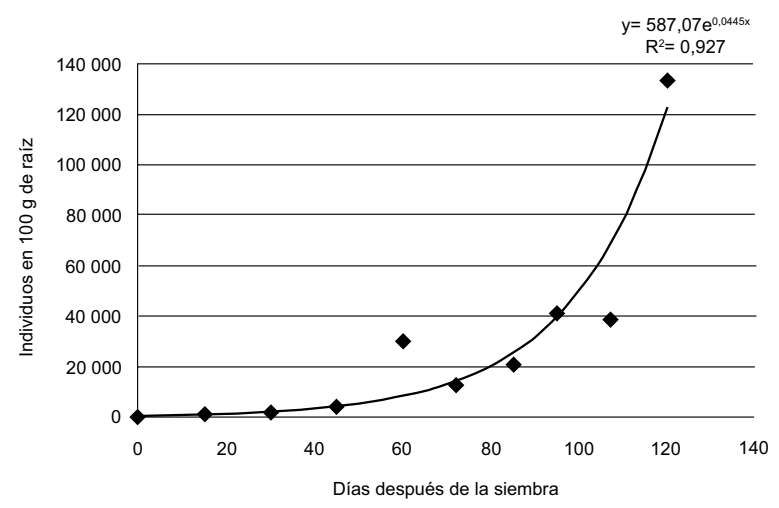

Figura 6. Progresión poblacional de Meloidogyne en raíz de arroz según edad de la plantación en la Región Huetar Atlántica, Costa Rica, en el periodo 20062009.

a diferencia de Pratylenchus, Meloidogyne tuvo un incremento poblacional exponencial, el incremento en la densidad de población de Meloidogyne también pudo explicarse por una función de regresión exponencial, que contribuiría a desarrollar un programa de manejo integrado del nematodo.

El crecimiento exponencial de Meloidogyne podría implicar que al final del ciclo de cultivo la densidad poblacional del mismo podría incrementarse a niveles que podrían resultar dañinos para la plantación. Se debe considerar que la fuente de alimento se convierte 
en un recurso limitado conforme crece la población o por antagonismo con otros nematodos.

Incidencia de los géneros de mayor importancia económica en el cultivo del arroz en las regiones Huetar Norte y Huetar Atlántica, de Costa Rica

Los respectivos niveles de incidencia de los nematodos o rangos de conteo para los géneros
Pratylenchus y Meloidogyne, se muestran en los Cuadros 2, 3, 4 y 5, la incidencia es estimada de acuerdo a la cantidad de individuos en $100 \mathrm{~g}$ de raíz, de acuerdo a cada modelo estimado y en función de los días de siembra en cada caso respectivamente. En dichos niveles de incidencia se puede observar que para Pratylenchus en la región Huetar Norte los valores o rangos de conteo son mayores, superando los 87000 individuos en niveles de incidencia 9 a los

Cuadro 2. Niveles de incidencia para la estimación de nematodos en función de los días de siembra (individuos en 100 g de raíz) del género Pratylenchus en raíz de arroz, en la Región Huetar Norte de Costa Rica. Generada en el periodo 2006-2009.

\begin{tabular}{|c|c|c|c|c|c|c|}
\hline & \multicolumn{6}{|c|}{ Región Huetar Norte } \\
\hline & \multicolumn{6}{|c|}{ Días después de la siembra } \\
\hline \multirow{11}{*}{ 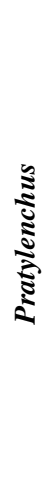 } & Escala & $0-28$ & 29-59 & $60-85$ & 86-107 & mayor a 107 \\
\hline & 0 & $\leq 80$ & $\leq 1000$ & $\leq 3000$ & $\leq 4700$ & $\leq 6000$ \\
\hline & 1 & $81-160$ & $1000-1600$ & $3001-4300$ & $4701-7000$ & $6001-8800$ \\
\hline & 2 & $161-1200$ & $1601-2300$ & $4301-8000$ & $7001-8500$ & $8801-11600$ \\
\hline & 3 & $1201-2300$ & $2301-3000$ & $8001-11500$ & $8501-10000$ & $11601-14500$ \\
\hline & 4 & $2301-3800$ & $3001-5500$ & $11501-16700$ & $10001-15500$ & $14501-22000$ \\
\hline & 5 & $3801-4600$ & $5501-6700$ & $16701-21000$ & $15501-18500$ & $22001-25800$ \\
\hline & 6 & $4601-5400$ & $6701-8000$ & $21001-24000$ & $18501-21300$ & $25801-30000$ \\
\hline & 7 & $5401-7000$ & $8001-14700$ & $24001-38000$ & $21301-39300$ & $30001-58000$ \\
\hline & 8 & $7001-8600$ & $14701-21600$ & $38001-52000$ & $39301-57000$ & $58001-87000$ \\
\hline & 9 & $\geq 8601$ & $\geq 21601$ & $\geq 52001$ & $\geq 57001$ & $\geq 87001$ \\
\hline
\end{tabular}

Cuadro 3. Niveles de incidencia para la estimación de nematodos en función de la fecha de siembra (individuos en $100 \mathrm{~g}$ de raíz) del género Meloidogyne en raíz de arroz, en la Región Huetar Norte de Costa Rica. Generada en el periodo 2006-2009.

\begin{tabular}{|c|c|c|c|c|c|c|}
\hline & \multicolumn{6}{|c|}{ Región Huetar Norte } \\
\hline & \multicolumn{6}{|c|}{ Días después de la siembra } \\
\hline \multirow{11}{*}{ 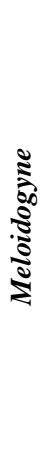 } & Escala & $\mathbf{0 - 2 8}$ & $29-59$ & $60-85$ & $86-107$ & mayor a 107 \\
\hline & 0 & $\leq 6$ & $\leq 30$ & $\leq 15$ & $\leq 130$ & $\leq 750$ \\
\hline & 1 & $7-11$ & $31-45$ & $16-30$ & $131-250$ & $751-1380$ \\
\hline & 2 & $12-19$ & $46-65$ & $31-105$ & $251-850$ & $1381-3500$ \\
\hline & 3 & $20-27$ & $66-82$ & $106-180$ & $851-1440$ & $3501-5780$ \\
\hline & 4 & $28-35$ & $83-235$ & $181-510$ & $1441-5300$ & $5781-14100$ \\
\hline & 5 & $36-40$ & $236-315$ & $511-675$ & $5301-7200$ & $14101-18350$ \\
\hline & 6 & $41-45$ & $316-400$ & $676-845$ & $7201-9200$ & $18351-22550$ \\
\hline & 7 & $46-49$ & $401-1750$ & $846-5700$ & $9201-3800$ & $22551-57100$ \\
\hline & 8 & $50-55$ & $1751-3100$ & $5701-10600$ & $38001-67000$ & $57101-91700$ \\
\hline & 9 & $\geq 56$ & $\geq 3101$ & $\geq 10601$ & $\geq 67001$ & $\geq 91700$ \\
\hline
\end{tabular}


Cuadro 4. Nivel de incidencia para la estimación de nematodos en función de los días de siembra (individuos en 100 g de raíz) del género Pratylenchus en raíz de arroz, en la Región Huetar Atlántica de Costa Rica. Generada en el periodo 2006-2009.

\begin{tabular}{|c|c|c|c|c|c|c|}
\hline & \multicolumn{6}{|c|}{ Región Huetar Atlántica } \\
\hline & \multicolumn{6}{|c|}{ Días después de la siembra } \\
\hline \multirow{11}{*}{ 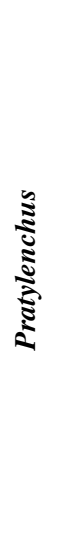 } & Escala & 0-28 & $29-59$ & $60-85$ & 86-107 & mayor a 107 \\
\hline & 0 & $\leq 72$ & $\leq 350$ & $\leq 1700$ & $\leq 1400$ & $\leq 4000$ \\
\hline & 1 & $73-140$ & $351-540$ & $1701-2400$ & $1401-2000$ & $4001-6400$ \\
\hline & 2 & $141-400$ & $541-780$ & $2401-3400$ & $2001-3000$ & $6401-8000$ \\
\hline & 3 & $401-640$ & $781-1000$ & $3401-4300$ & $3001-4000$ & $8001-9800$ \\
\hline & 4 & $641-1500$ & $1001-1400$ & $4301-5500$ & $4001-10000$ & $9801-13000$ \\
\hline & 5 & $1501-2000$ & $1400-1600$ & $5501-6000$ & $10001-13000$ & $13001-14700$ \\
\hline & 6 & $2001-2500$ & $1601-2000$ & $6001-6700$ & $13001-16000$ & $14701-16500$ \\
\hline & 7 & $2501-2750$ & $2000-8000$ & $6701-16000$ & $16001-30000$ & $16501-32000$ \\
\hline & 8 & $2750-3000$ & $8001-14000$ & $16001-25000$ & $30001-42000$ & $32001-47000$ \\
\hline & 9 & $\geq 3001$ & $\geq 14001$ & $\geq 25001$ & $\geq 42001$ & $\geq 47001$ \\
\hline
\end{tabular}

Cuadro 5. Nivel de incidencia para la estimación de nematodos en función de los días de siembra (individuos en 100 g de raíz) del género Meloidogyne en raíz de arroz, en la Región Huetar Atlántica de Costa Rica. Generada en el periodo 2006-2009.

\begin{tabular}{|c|c|c|c|c|c|c|}
\hline & \multicolumn{6}{|c|}{ Región Huetar Atlántica } \\
\hline & \multicolumn{6}{|c|}{ Días después de la siembra } \\
\hline & Escala & $0-28$ & 29-59 & $60-85$ & $86-107$ & mayor a 107 \\
\hline \multirow{10}{*}{ 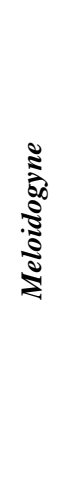 } & 0 & $\leq 11$ & $\leq 43$ & $\leq 1000$ & $\leq 1600$ & $\leq 2700$ \\
\hline & 1 & $12-22$ & $44-75$ & $1001-1750$ & $1601-2850$ & $2001-5000$ \\
\hline & 2 & $23-35$ & $76-130$ & $1751-4800$ & $2851-6300$ & $5001-10000$ \\
\hline & 3 & $36-45$ & $131-180$ & $4801-7800$ & $6301-10000$ & $10001-15000$ \\
\hline & 4 & $46-65$ & $181-450$ & $7801-14000$ & $10001-20600$ & $15001-26000$ \\
\hline & 5 & $66-75$ & $451-580$ & $14001-17000$ & $20601-26000$ & $26001-31000$ \\
\hline & 6 & $76-85$ & $581-700$ & $17001-20000$ & $26001-31500$ & $31001-36000$ \\
\hline & 7 & $86-800$ & $701-5200$ & $20001-75000$ & $31501-50500$ & $36001-92000$ \\
\hline & 8 & $801-1700$ & $5201-9700$ & $75001-132000$ & $50501-69500$ & $92001-150000$ \\
\hline & 9 & $\geq 1701$ & $\geq 9701$ & $\geq 132001$ & $\geq 69501$ & $\geq 150001$ \\
\hline
\end{tabular}

107 días, mientras que en la región Huetar Atlántica los niveles de incidencia son de 9 y para los 107 días después de la siembra, los rangos de conteo llegaron a 47001 individuos lo que nos indica que la incidencia de Pratylenchus fue mayor en la Región Huetar Norte que en la Región Huetar Atlántica.
En cuanto a Meloidogyne, este género muestra valores o rangos de conteo menores en la Región Huetar Norte, los valores, a diferencia de Pratylenchus, muestran un incremento exponencial, por lo que Meloidogyne alcanzó 91700 individuos en el nivel de incidencia 9 a los 107 días (Cuadro 4). Por otro lado en la Región 
Huetar Atlántica, los valores o rangos de conteo son mayores, alcanzando densidades poblacionales mayores a 150001 individuos para el nivel 9 a los 107 días después de la siembra, esto nos indica que la incidencia de Meloidogyne fue mayor para la Región Huetar Atlántica que para la Región Huetar Norte (Cuadro 5).

Los resultados obtenidos en este estudio, concuerdan con los reportados por la literatura para otras regiones en Costa Rica, tanto por los géneros detectados, como por la importancia relativa de cada género.

Los géneros de nematodos de mayor importancia detectados en el cultivo del arroz en las regiones Huetar Norte y Huetar Atlántica de Costa Rica fueron Pratylenchus, Meloidogyne y Helicotylenchus. Aunque por la densidad en la que fueron detectados, y por ser reconocidas plagas en otros cultivos, Pratylenchus y Meloidogyne son los géneros que pudieran ser de importancia económica en el cultivo del arroz en estas regiones.

Estudiando la frecuencia de presencia de los géneros detectados y su densidad de población por región, se infiere un posible antagonismo entre Pratylenchus y Meloidogyne.

Tanto Pratylenchus como Meloidogyne mostraron una correlación alta entre el aumento de su densidad poblacional y la edad de la plantación de arroz, además Pratylenchus tuvo un crecimiento poblacional lineal, mientras que Meloidogyne mostró un crecimiento poblacional exponencial. Ambos modelos matemáticos y sus respectivos niveles de incidencia para la estimación en función de los días de siembra, serán de gran utilidad al desarrollar un plan de manejo integrado de plagas para ambos nematodos, esto porque permitirán predecir el comportamiento de las poblaciones con el fin de determinar qué tipo de control es más adecuado en un momento dado.

Se recomienda en futuros trabajos relacionados con la determinación de incidencia, calcular el umbral de daños de estos organismos sobre el arroz, o determinar el nível mínimo y máximo de pérdidas sobre indicadores del crecimiento de la planta o de rendimiento.

\section{LITERATURA CITADA}

Esquivel, A. 1996. Algunas técnicas de extracción de nematodos. Boletín Agrario N ${ }^{\circ} 56$ Año 15, Escuela de Ciencias Agrarias, Universidad Nacional, Heredia. $21 \mathrm{p}$.

Fernández, M; Ortega, J. 1982. Comportamiento de las poblaciones de nematodos fitoparásitos en plátano enano Cavendish. Ciencias de la Agricultura 13:7-17.

González, L. 1978. Nematodos fitoparásitos asociados con la rizosfera de arroz y maíz en varias zonas agrícolas de Costa Rica. Agronomía Costarricense 2(2):171-173.

López, JD. 2006. Determinación preliminar de géneros y densidades poblacionales de nematodos asociados al cultivo del arroz (Oryza sativa) en la región Huetar Norte de Costa Rica. Proyecto Bach. Ing. Agr. San Carlos, CR, ITCR. 52 p.

López, R; Salazar, L; Azofeifa, J. 1987. Nematodos asociados al arroz (Oriza sativa L.) en Costa Rica. V. Frecuencia y densidades poblacionales en las principales zonas productoras. Agronomía Costarricense. 11(2):215-220.

Ramírez, J. 2001. Comportamiento agronómico, productivo e industrial de arroz (Oriza sativa) variedad SETESA9 en La Vega, San Carlos, Costa Rica. Informe Bach. Ing. Agr. San Carlos, Costa Rica. ITCR. 69 p.

Sancho, C; Salazar, L. 1985. Nematodos parásitos del arroz (Oriza sativa L.) en el sureste de Costa Rica. Agronomía Costarricense 9(2):161-163.

Sancho, C; Salazar, L; López, R. 1987. Efecto de la densidad inicial del inóculo sobre la patogenicidad de Meloidogyne salasi en tres cultivares de arroz. Agronomía Costarricense 11(2):233-238.

Suárez, H; Rosales, L. 2004. Competition between the plant-parasitic nematodes Pratylenchus neglectus and Meloidogyne chitwoodii. Journal of Nematology 26(3):286-295.

Umesh, KC. 1994. Competition between the plant-parasitic nematodes Pratylenchus neglectus and Meloidogyne chitwoodi. Journal of Nematology 26(3):286-295. 